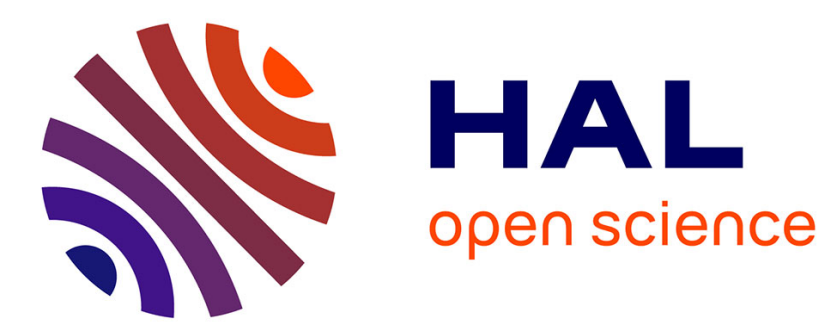

\title{
Variable genetic element typing: a quick method for epidemiological subtyping of
}

K. Pannier, K. Heuner, C. Lück

\section{To cite this version:}

K. Pannier, K. Heuner, C. Lück. Variable genetic element typing: a quick method for epidemiological subtyping of. European Journal of Clinical Microbiology and Infectious Diseases, 2010, 29 (4), pp.481487. 10.1007/s10096-009-0872-8 . hal-00568348

\section{HAL Id: hal-00568348 \\ https://hal.science/hal-00568348}

Submitted on 23 Feb 2011

HAL is a multi-disciplinary open access archive for the deposit and dissemination of scientific research documents, whether they are published or not. The documents may come from teaching and research institutions in France or abroad, or from public or private research centers.
L'archive ouverte pluridisciplinaire $\mathbf{H A L}$, est destinée au dépôt et à la diffusion de documents scientifiques de niveau recherche, publiés ou non, émanant des établissements d'enseignement et de recherche français ou étrangers, des laboratoires publics ou privés. 
Diseases

Editorial Manager(tm) for European Journal of Clinical Microbiology \& Infectious

Manuscript Draft

Manuscript Number: EJCMID-D-09-00409R1

Title: Variable Genetic Element Typing: A quick method for epidemiological subtyping of Legionella pneumophila

Article Type: Brief Report

Keywords: Legionella pneumophila; genetic typing; epidemiology

Corresponding Author: Dr. med. Christian Lueck,

Corresponding Author's Institution:

First Author: Kristin Pannier

Order of Authors: Kristin Pannier; Klaus Heuner, Dr.; Christian Lück, Dr.; Christian Lueck

Abstract: A total of 57 isolates of L. pneumophila were randomly selected from the German National Legionella strain collection and typed by monoclonal antibody subgrouping, seven gene locus sequence-based typing (SBT) scheme and a newly developed variable element typing (VET) system based on the presence or absence of ten variable genetic elements. These elements were detected while screening of a genomic library of strain Corby as well as taken from published data for PAI-1 (pathogenicity island) from strain Philadelphia. Specific primers were designed and used in gel based PCRs. PCR amplification of the mip gene that served as a control. The endpoint was the presence / absence of a PCR product on an ethidiumbromide strained gel. In the present study the index of discrimination was somewhat lower than that of the SBT ( 0.87 versus 0.97$)$. Nevertheless, the results obtained showed as a 'proof of principle' that this simple and quick typing assay might be useful for the epidemiological characterization of Legionella pneumophila strains. 
1 Concise Article

2 Variable Genetic Element Typing: A quick method for

3 epidemiological subtyping of Legionella pneumophila

4

$5 \quad$ K. Pannier, K. Heuner, C. Lück

6

$7 \quad$ K. Pannier, C. Lück

8 Institute of Medical Microbiology and Hygiene, TU Dresden, 01307 Dresden, Germany

9

K. Heuner

11 Robert Koch-Institut, Nordufer 20, P26 - Nosocomial Infections of the Elderly, 13353 Berlin,

12 Germany

13

14

15

16 Corresponding author:

17 C. Lück

18 Institute of Medical Microbiology and Hygiene, TU Dresden,

19 Fiedlerstraße 42,

20 Dresden, D- 01307, Germany

21 E-mail: Christian.Lueck@tu-dresden.de

23 European Journal of Clinical Microbiology and Infectious Diseases, 2009 


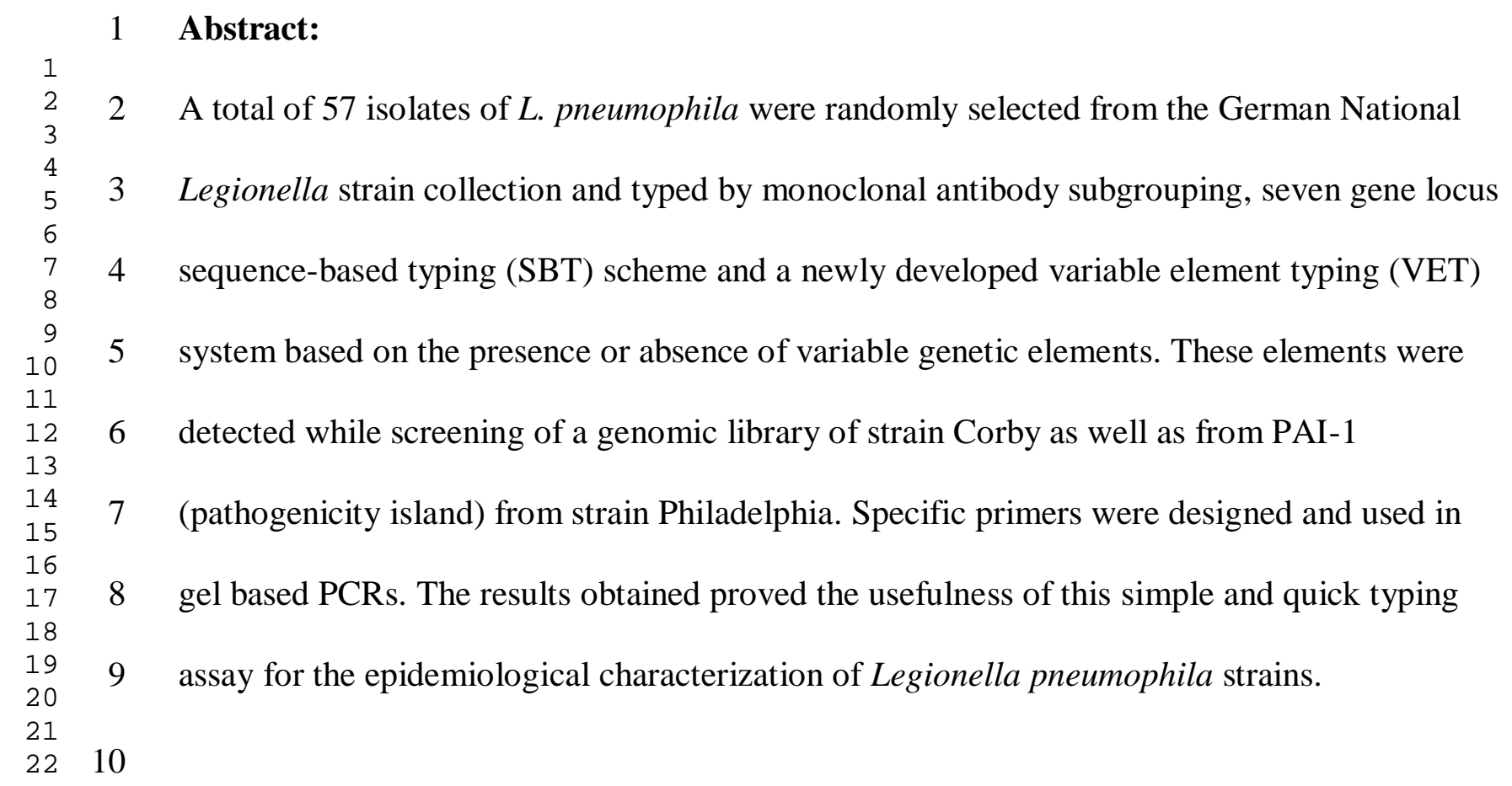




\section{Introduction}

2 Members of the genus Legionella are Gram-negative bacteria and normally occupy natural

3 aquatic environments where they survive as intracellular parasites of protozoa. Currently the

4 genus Legionella contains more than 50 species. Among them the species L. pneumophila is

5 the most frequent [1;2]. L. pneumophila serogroup (sg) 1 is the most common cause of

6 pneumonia occurring as sporadic or endemic disease that may be acquired from different

7 environmental sources [3]. For public health authorities it is essential to detect the source of

8 infection promptly by comparing clinical and environmental strains of L. pneumophila to

9 conduct decontamination measures and to prevent further cases. For this numerous

10 phenotypic and genotypic typing methods have been applied to the epidemiological typing of

11 L. pneumophila in the recent years [4]. This include monoclonal antibody (MAb)

12 subgrouping as a rapid screening method [3]and genotyping methods such as amplified

13 fragment length polymorphism (AFLP) [4], pulsed-field gel electrophoresis (PFGE) [4;5] and 14 sequence based typing [6-8]. In contrast to band based typing methods that are difficult to 15 standardize the developed sequence based typing (SBT) has the potential of excellent 16 typeability, interlaboratory reproducibility and epidemiologic concordance[9]. However the

17 SBT is rather expensive and might therefore not applicable to all strains and in all

18 laboratories. The specific gene content of each strain sequenced so far is constituted of 7-11\% 19 of each genome [10-12]. In addition, variable elements/ pathogenicity island are present in 20 some strains [13].

21 The purpose of this study was to investigate the usefulness of a binary typing system (VET) 22 based on the presence/ absence of defined genetic elements by using gel based PCR. These 23 data were compared to the European Working Group on Legionella Infections (EWGLI) SBT 24 system. 


\section{$1 \quad$ Materials and Methods}

2 Legionella pneumophila strains. A total of 57 isolates of Legionella pneumophila were

3 randomly selected from the Dresden strain collection including the strains from which the

4 complete genomes are published. From strain Corby we used a panel of variants (mutants)

5 (EUL 135 to 139) that differ in their ability to multiply in amoebae and macrophages showed

6 different reactivity patterns with MAbs due to a mutation in a lipopolysaccharide synthesis

7 gene or that were resistant to rifampicin [7]. 40 strains were by definition unrelated to any

8 other isolates in the study, i.e. they were isolated from sporadic cases or if they were part of

9 an outbreak or cluster of legionellosis only one isolate was included in the analysis. 32 isolates were obtained during epidemiological studies (Table 1) and comprise twelve sets of related strains (A to M). Some of them were indistinguishable by MAb subgrouping and SBT

12 thus proving a transmission from the environmental source to the patient. Sets $\mathrm{C}$ and $\mathrm{H}$

13 differed in both markers, whereas sets $\mathrm{K}, \mathrm{L}$, and $\mathrm{M}$ were indistinguishable by genetic typing 14 but expressed a different monoclonal subtype which is related to the deletion of the lag -1 15 gene [5] (Table 1).

17 Serotyping and sequence based typing (SBT)

18 Serological typing of L. pneumophila strains was performed by using the Dresden panel of monoclonal antibodies and the results were published previously [3;5;8]. Genotyping was performed by using the seven genes SBT [7;8]. Briefly, seven gene targets comprising flaA, pile, asd, mip, momp, proA and neuA were amplified by PCR and sequenced. The assignment of the sequence type (ST) was carried out by using SBT database or the online sequence type checker (http://www.hpa-bioinfotools.org.uk/legionella/php/sbt_query1.php ).

Variable element typing (VET). Target sequences for variable element typing were selected from a genomic library of strain Corby according to Heuner et al. [14] with minor modifications. Briefly, chromosomal DNA of L. pneumophila Corby (sg 1) was partially 
1 digested with Sau3A. Fragments ranging from 1.0 to $4.0 \mathrm{~kb}$ were ligated into the BamHI

2 restriction site of vector pUC19 and transformed into E. coli DH5 $\alpha$. The DNA sequence of

3 the inserted Corby-DNA was determined by using standard $\mathrm{m} 13$ primers and primer walking.

4 Fragments that could not be aligned to the Philadelphia genome available at that time were

5 selected. Primer specific for these genetic elements were designed and used for typing (Table

6 2). For $m a g \mathrm{~A}$ and $\operatorname{tra\mathrm {D}}$ sequences from the pathogenicity island [13] were used.

7 DNAs from L. pneumophila strains was prepared as for sequenced based typing [7]. The

8 VET- PCR were performed with 10pmol of each primer, 10 $\mu 1$ of DNA-puffer, 5U of Gold-

9 Taq-Polymerase, $2 \mu 1$ DNA of each strain, and 200pmol of each dNTP at a final volumes of

$1050 \mu 1$. The annealing temperature was $58^{\circ} \mathrm{C}$. Gel electrophoresis was performed on $1 \%$

11 agarose gels. Gels were stained with ethidium bromide and analyzed under UV-light. PCR

12 products of the expected size were considered positive. At the beginning of these experiments

13 the specificity of the PCR products was confirmed by DNA sequencing [7]. In each run the 14 mip gene PCR of the SBT scheme were used as a positive control [7]. All VET PCR assays 15 were run at least twice.

16 A VET-index was applied as the summary of the binary results (presence/absence of a PCR 17 product). Hereby, these results of each three PCR assays were put together. If a band was 18 identified on the gel, the first PCR received the number 1; the second get the number 2 and 19 the third the number 4. If no PCR product was obtained the number 0 was assigned.

20 As a result each set of the three PCR assays could be summarized as one digit between 0 and 21 7. All ten PCR assays could therefore presented as a 4 digit number. The advantage of this 22 VET-index is a reduced size, a more clearly arrangement and the possibility to exchange these 23 data between laboratories. 


\section{Results and Discussion}

2 The primary aim of this study was to evaluate the utility of VET as a genotyping method for

3 L. pneumophila. The primers developed and used in this study were aligned to the four

4 available complete genomes of $L$. pneumophila $[10 ; 11 ; 12]$ by using the Basic Local

5 Alignment Search Tool (BLAST) available online (www.ncbi.nlm.nih.gov/BLAST) [15]. The

6 results of this search demonstrated that the binding sites of some primers were present some

7 of the four genomes. The investigation of the strains Philadelphia-1, Lens, Paris and Corby by

8 using the new VET-PCRs confirmed the expected results, i. e. all PCR product predicted by

9 the BLAST search could readily detected by using the ten VET-PCR assays. The variable

10 elements used in this typing assay are relatively randomly distributed in the genome of the

11 strains from which complete sequenced genomes are available (Fig.1).

12 In addition, all five variants of the Corby strains that differ in the cultivability in amoebal

13 hosts, point mutations in the $r p o \mathrm{~B}$ and the lag-1 genes showed the same VET results (data not

14 shown) thus arguing that the markers detected in the VET are stable as were the SBT results

15 [7].

16 For 57 of the strains investigated by using variable element typing (VET) results were

17 obtained for 51. In six strains none of the elements could be detected by the current assays

18 (Tab. 1, Fig.2). Consequently the VET index of these strains was 0000. Since the positive 19 control PCR using the mip primers of the SBT scheme gave positive results we are sure that 20 the genetic elements or at least one of the primer binding sites were readily absent (or to less 21 identical). From a total of 57 strains, 32 related strains were merged into 12 sets (A to M) 22 (Table 1, Fig 2). From these sets of related strains the results of seven sets (A, B, D, E, F, G, 23 and J) showed that related strains were indistinguishable by using for MAb typing, SBT and 24 VET. For further two sets $(\mathrm{C}$ and $\mathrm{H})$, the serotype/MAb subtype matched. However, SBT and 25 VET of the environmental isolates were different from that of the patient's isolates. Vice 26 versa three sets (K, L, and $\mathrm{M})$ showing the same genetic fingerprints by using SBT and VET 
1 were distinguishable by MAb typing. It has been shown previously that changes in genes

2 involved in the LPS synthesis might change the reactivity with MAbs. Thus the loss of the

3 lag-1 gene coding for an O-acetyltransferase is responsible for the switch from MAbtype

4 Philadelphia/ Knoxville to the MAb type OLDA/Denver [5;16;17].

5 Taking into account the moderate number of unrelated strains $(n=40)$ in our study an

6 moderate index of discrimination (IOD) of 0.87 was calculated. This is lower than that

7 published previously for SBT, but higher than that for MAb typing and AFLP [4;7].

8 Interestingly, in some cases strains belonging to different serotypes were indistinguishable by

9 VET. For example, the most frequently occurring VET-Index 0250 was found in several

10 strains belonging to different serogroups/ MAb-types and SBT variants. Thus strains Uppsala

11 21, Uppsala 59, L04-567 or L04-564 showed different serotypes/monoclonal subtypes like

12 OLDA, Philadelphia or serogroup 10. In addition, strains with the VET-Index 0251 (e.g., 13 L02-298, L05-129) or VET-Index 4411 (e.g., Heidelberg P1, P9) belonged to different 14 serogroups (Table 1).

15 Due to the limited number of strains a general conclusion concerning the correlation between

16 these two genotyping methods is difficult. It is not surprising that a complete correlation does 17 not exist between both genotyping methods, since SBT detects variations in seven genes 18 belonging to the core genome whereas VET detects the absence or presence of variable 19 genetic elements like PAIs. Therefore, it is not surprising that some strains of sequence type 20 (ST) 1 investigated here, had a different VET-Index.

21 SBT is a powerful tool for subtyping L. pneumophila strains and is currently the gold standard 22 within the European Working Group on Legionella Infections (EWGLI). Data obtained in 23 different countries can easily be compared and the European data base currently containing 24 hundreds of types can be used both in epidemiological investigations and to assess the risk 25 associated with environmental reservoirs that are contaminated with illness-associated strains. 26 However SBT is relatively expensive and therefore might not be applicable to all laboratories. 
1 The aim of the present study was to evaluate an alternative rapid and cheep PCR approach

2 based on the absence or presence of defined genetic elements in different strains. A recent

3 study from Australia suggested that in all strains a similar repertoire of virulence gene exist

4 [18]. In accordance with this study our results demonstrate that the presence of the $l v h$ locus,

5 the $t r b-1$ locus and the pathogenicity island I is variable in different strains. So far no

6 association of the presence of these genetic elements with the putative virulence i. e. the

7 origin of the strains could be demonstrated.

8 The usefulness of the VET approach as a technique for subtyping of strains belonging to

9 different serogroups of L. pneumophila was clearly demonstrated. The possibility to express

10 the data as binary codes allows the standardization and portability of these results as shows

11 for Staphylococcus aureus and Escherichia coli [19;20]. It remains an open question and 12 needs to be experimentally proven with a larger number of strains to whether the use of VET 13 is indeed suitable for the epidemiological subtyping of L. pneumophila. Finally, it must be 14 pointed out that these genetic elements might serve as candidates for a DNA chip based 15 typing system similar to that for St. aureus [21]. In summary, our current results are 16 encouraging. The VET scheme showed good typeability and discriminatory power for $L$. 17 pneumophila. However, further variable elements must be identified to increase both, the 18 typeability and the discriminatory potential.

20 Acknowledgements. Legionella-strains were kindly supplied by C. Aepinus (Würzburg), W. 21 Ehret (Augsburg), E. Halle (Berlin), Hohlfelder (Heidelberg), D. Jonas (Freiburg), W. 22 Matthys (Münster), R. Pfüller (Berlin), R. Marre (Ulm), C. Schoerner (Erlangen), K. Weist 23 (Berlin). We are grateful to Kerstin Lück and Jutta Paasche for technical assistance. 
Table 1. Legionella pneumophila strains

\begin{tabular}{|c|c|c|c|c|c|}
\hline Strain designation & $\begin{array}{l}\text { Serogroup, } \\
\text { Monoclonal } \\
\text { Subtype }\end{array}$ & $\begin{array}{l}\text { Sequence } \\
\text { Type } \\
\text { (ST) }\end{array}$ & $\begin{array}{l}\text { Allels profile of } \\
\text { flaA, pilE, asd, } \\
\text { mip, ompS, proA } \\
\text { and neuA }\end{array}$ & $\begin{array}{l}\text { VET- } \\
\text { Index }\end{array}$ & Origin of strains \\
\hline Lens & 1 Benidorm & 15 & $12,9,26,5,26,17,15$ & 0210 & Clinical isolate community acquired pneumonia, unrelated \\
\hline Augsburg-1 & 1 Benidorm & 42 & $4,7,11,3,11,12,9$ & 7331 & Clinical isolate community acquired pneumonia, unrelated \\
\hline Berlin 11 & 1 Benidorm & 425 & $2,10,3,15,9,4,11$ & 0240 & Clinical isolate nosocomial pneumonia, unrelated \\
\hline L02-287 & 1 Benidorm & 181 & $3,7,1,12,14,9,9$ & 3011 & Clinical isolate community acquired pneumonia, unrelated \\
\hline L02-298 & 1 Benidorm & 42 & $4,22,11,3,11,12,9$ & 0251 & Clinical isolate community acquired pneumonia, unrelated \\
\hline L04-412 & 1 Knoxville & 9 & $3,10,1,3,14,9,11$ & 0041 & Clinical isolate travel-associate pneumonia, unrelated \\
\hline Corby & 1 Knoxville & 51 & $6,10,15,28,9,14,6$ & 7341 & Clinical isolate community acquired pneumonia, unrelated \\
\hline L01-443 & 1 Knoxville & 9 & $3,10,1,3,14,9,11$ & 6141 & Clinical isolate nosocomial pneumonia, unrelated \\
\hline Berlin 4 & 1 OLDA & 1 & $1,4,3,1,1,1,1$ & 2740 & Clinical isolate nosocomial pneumonia, unrelated \\
\hline Charite $16297 / 2000$ & $1 \mathrm{OLDA}$ & 1 & $1,4,3,1,1,1,1$ & 6650 & Clinical isolate community acquired pneumonia, unrelated \\
\hline Goettingen $12 / 98$ & 1 OLDA & 1 & $1,4,3,1,1,1,1$ & 0650 & Clinical isolate community acquired pneumonia, unrelated \\
\hline L03-610 & 1 OLDA & 7 & $1,4,3,1,1,1,6$ & 0240 & Clinical isolate community acquired pneumonia, unrelated \\
\hline L04-567 & $1 \mathrm{OLDA}$ & 1 & $1,4,3,1,1,1,1$ & 0250 & Clinical isolate nosocomial pneumonia, unrelated \\
\hline Paris & 1 Philadelphia & 1 & $1,4,3,1,1,1,1$ & 0050 & Clinical isolate community acquired pneumonia, unrelated \\
\hline Philadelphia & 1 Philadelphia & 36 & $3,4,1,1,14,9,1$ & 0430 & Clinical isolate community acquired pneumonia, unrelated \\
\hline L04-280 & 1 Philadelphia & 23 & $2,3,9,10,2,1,6$ & 7340 & Clinical isolate travel-associate pneumonia, unrelated \\
\hline L02-034 & 10 & 440 & $3,10,1,28,14,9,1$ & 0000 & Clinical isolate nosocomial pneumonia, unrelated \\
\hline L02-303-1 & 15 & 337 & $10,22,7,28,16,18,6$ & 2011 & Clinical isolate nosocomial pneumonia, unrelated \\
\hline Heidelberg P9 & 4 Portland & 67 & $1,10,3,28,9,4,1$ & 4411 & Clinical isolate nosocomial pneumonia, unrelated \\
\hline W 01/1993 & 5 Cambridge & 245 & $1,10,3,13,9,4,1$ & 7310 & Environmental isolate, unrelated \\
\hline
\end{tabular}




\begin{tabular}{|c|c|c|c|}
\hline Chicago 2 & 6 Chicago & 30 & $3,10,1,3,14,9,9$ \\
\hline Finnl. 10 & 6 Chicago & 68 & $3,13,1,28,14,9,3$ \\
\hline Heidberg P1 & 6 Dresden & 330 & $7,10,17,3,4,11,3$ \\
\hline L04-507 & 1 Philadelphia & 332 & $7,10,17,6,14,11,3$ \\
\hline L04-506 & 1 Philadelphia & 332 & $7,10,17,6,14,11,3$ \\
\hline W05-007 & 6 Chicago & 424 & $7,10,17,3,13,14,9$ \\
\hline L04-597 & 6 Chicago & 424 & $7,10,17,3,13,14,9$ \\
\hline L05-008 & 6 Chicago & 424 & $7,10,17,3,13,14,9$ \\
\hline W04-952 & $1 \mathrm{OLDA}$ & 1 & $1,4,3,1,1,1,1$ \\
\hline W04-954 & 1 OLDA & 1 & $1,4,3,1,1,1,1$ \\
\hline W04-956 & 1 OLDA & 1 & $1,4,3,1,1,1,1$ \\
\hline Erl. 361/1 & $1 \mathrm{OLDA}$ & 1 & $1,4,3,1,1,1,1$ \\
\hline L04-541 & 1 OLDA & 1 & $1,4,3,1,1,1,1$ \\
\hline W05-67-1 & 1 Benidorm & 426 & $2,6,17,14,12,11,11$ \\
\hline W05-67-4 & 1 Benidorm & 426 & $2,6,17,14,12,11,11$ \\
\hline L04-497 & 1 Benidorm & 407 & $6,10,15,3,21,14,9$ \\
\hline L97-229/1 & 1 Philadelphia & 387 & $2,6,17,14,13,11,11$ \\
\hline L97-229/2 & 1 Philadelphia & 387 & $2,6,17,14,13,11,11$ \\
\hline W04-989 & 10 & 245 & $2,10,3,13,9,4,1$ \\
\hline W04-990 & 10 & 245 & $2,10,3,13,9,4,1$ \\
\hline
\end{tabular}

Clinical isolate nosocomial pneumonia, unrelated

Clinical isolate community acquired pneumonia, unrelated

Clinical isolate nosocomial pneumonia, unrelated

Clinical isolate community acquired pneumonia, unrelated

Clinical isolate community acquired pneumonia, unrelated

Set A: Hospital water isolate

Set A: Patients isolate nosocomial pneumonia

Set A: Patients isolate nosocomial pneumonia

Set B: Hospital water isolate

Set B: Hospital water isolate

Set B: Hospital water isolate

Set B: Patients isolate nosocomial pneumonia

Set B: Patients isolate nosocomial pneumonia

Set C: Environmental isolate working place

Set $\mathrm{C}$ : Environmental isolate working place

Set C: Patients isolate community-acquired pneumonia

Set D: Patients isolate community-acquired pneumonia

Set D: Patients isolate community-acquired pneumonia

Set E: Environmental isolate

Set E: Environmental isolate 


\begin{tabular}{|c|c|c|c|c|}
\hline L04-564 & 10 & 245 & $2,10,3,13,9,4,1$ & 0010 \\
\hline L04-565 & 10 & 245 & $2,10,3,13,9,4,1$ & 0010 \\
\hline W05-192 & 10 & 246 & $2,10,3,28,9,4,11$ & 0010 \\
\hline W05-191 & 10 & 246 & $2,10,3,28,9,4,11$ & 0010 \\
\hline Koper 1 & 1 Knoxville & 146 & $1,10,18,10,2,1,6$ & 4251 \\
\hline L02-435 & 1 Knoxville & 146 & $1,10,18,10,2,1,6$ & 4251 \\
\hline W05-174-1 & 10 & 248 & $3,13,1,1,14,9,11$ & 0000 \\
\hline W05-177-1 & 10 & 248 & $3,13,1,1,14,9,11$ & 0000 \\
\hline L05-129 & 10 & 247 & $1,4,3,5,1,1,11$ & 0251 \\
\hline L01-127 & 1 OLDA & 1 & $1,4,3,1,1,1,1$ & 0250 \\
\hline W01/1967 & 1 OLDA & 1 & $1,4,3,1,1,1,1$ & 0250 \\
\hline Uppsala 3 & 1 Knoxville & 9 & $3,10,1,3,14,9,11$ & 0000 \\
\hline Uppsala 10 & 1 OLDA & 9 & $3,10,1,3,14,9,11$ & 0000 \\
\hline Uppsala 59 & 1 Philadelphia & 1 & $1,4,3,1,1,1,1$ & 0250 \\
\hline Uppsala 60 & 1 OLDA & 1 & $1,4,3,1,1,1,1$ & 0250 \\
\hline Uppsala 21 & 1 Philadelphia & 1 & $1,4,3,1,1,1,1$ & 0250 \\
\hline Uppsala 22 & $1 \mathrm{OLDA}$ & 1 & $1,4,3,1,1,1,1$ & 0250 \\
\hline
\end{tabular}

Set E: Patients isolate community-acquired pneumonia

Set E: Patients isolate community-acquired pneumonia

Set F: Hospital water isolate

Set F: Hospital water isolate

Set G: Environmental isolate from a hotel

Set G: Patients isolate travel-associated pneumonia

Set $\mathrm{H}$ : Environmental isolate

Set $\mathrm{H}$ : Environmental isolate

Set H: Patients isolate community-acquired pneumonia

Set J: Clinical isolate nosocomial pneumonia

Set J: Environmental isolate

Set K: Clinical isolate nosocomial pneumonia

Set K: Clinical isolate nosocomial pneumonia

Set L: Clinical isolate nosocomial pneumonia

Set L: Clinical isolate nosocomial pneumonia

Set M: Clinical isolate nosocomial pneumonia

Set M: Clinical isolate nosocomial pneumonia 
Table 2. Primers used for variable element typing (VET)

\begin{tabular}{|c|c|c|c|c|}
\hline NR & $\begin{array}{l}\text { Primer } \\
\text { designation }\end{array}$ & Sequence ( $\left.5^{\prime}-3^{\prime}\right)$ & $\begin{array}{l}\text { Size of the PCR } \\
\text { Product/ gene }\end{array}$ & Reference \\
\hline \multirow[t]{2}{*}{1} & TrbI1-F & TAA GGA GTG AGA CTG ATG AAT C & $1247 /$ trbII & 12 \\
\hline & TrbI1-R & CAT TGC ACT CCT CCT GTA TCA AT & & \\
\hline \multirow[t]{2}{*}{2} & TraG1-F & TGG AGA CGG TCA ATG AGC TTG A & $1279 /$ traG1 & 12 \\
\hline & TraG1-R & CAT ATC GCG AGC CGA TGC GCT G & & \\
\hline \multirow[t]{2}{*}{3} & MRR-F & AAT GAG AAG TCT TGG CTA TAC TTA & 835/lpc0219 & This study \\
\hline & MRR-R & GAT ATT CTC TAG CTT CGT TCG & & \\
\hline \multirow[t]{2}{*}{4} & $\mathrm{R} 33-\mathrm{F}$ & CGA CTA GGC GCT TAA TTC TTG & $830 / l p c 2786$ & This study \\
\hline & $\mathrm{R} 33-\mathrm{R}$ & ACG GAA CCA TTA AGA CAG GTT & & \\
\hline \multirow[t]{2}{*}{5} & F2-F & ACT GGC ATG AGC AAT CAG TAG & $1017 / p a c L$ & This study \\
\hline & F2-R & ATG ATC ACC TGT CAG GAC AGA & (lpc2098) & \\
\hline \multirow[t]{2}{*}{6} & TraD-F & GCT TAT CAT CAC TTG CCC TTT & $633 /$ traD & 13 \\
\hline & TraD-R & GCA GAG ATA CAC CAC CAA TCC GA & & \\
\hline \multirow[t]{2}{*}{7} & LvhB10-F & GCA ATC GGA CTC AGG TTG CTA & $710 / l v h B 10$ & 12 \\
\hline & LvhB10-R & CTG CCA AAG CGC TCG AAG AAA & & \\
\hline \multirow[t]{2}{*}{8} & MagA-F & CTC TAT CGC TAA CGC ACA AGG & $469 / m a g A$ & 13 \\
\hline & MagA-R & CGT TGA AGT AGT TAG TGA AAG & & \\
\hline \multirow[t]{2}{*}{9} & $127-2-\mathrm{F}$ & AAG CGA TTA GCA TAT TAC GGT TTC & 127/pacL & This study \\
\hline & $127-2-\mathrm{R}$ & CAT TGA TGA GAA CAA CTG CTA TTA TAA & & \\
\hline \multirow[t]{2}{*}{10} & $298-\mathrm{F}$ & GAT CTT TTA TGG CTG TTG TTT GAG C & 298/lpc1159-60 & This study \\
\hline & 298-2-R & GCC GCC TAT TTT TCG CAC TTT A & & \\
\hline
\end{tabular}




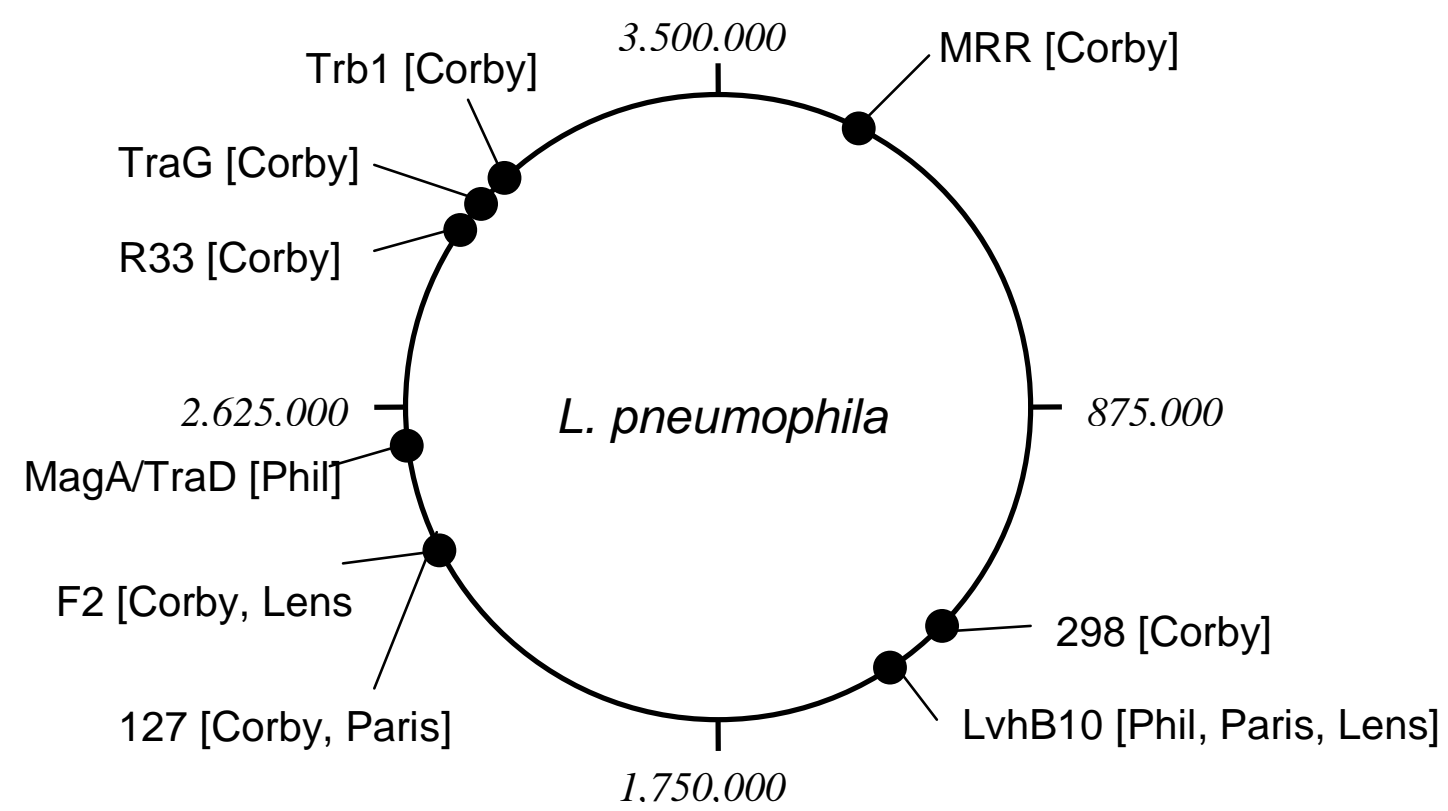

Fig. 1. Scheme of the location of the variable genetic elements in the four published genomes of L. pneumophila strains Philadelphia, Paris, Lens, and Corby 


\begin{tabular}{|c|c|c|}
\hline Sg/ MAb type & Set & ST \\
\hline 1 Knoxville & & 51 \\
\hline 1 Philadelphia & & 23 \\
\hline 1 Knoxville & & 9 \\
\hline 1 Beni & & 42 \\
\hline 5 Cambridge & & 245 \\
\hline 1 OLDA & & 1 \\
\hline 6 Chiccago & & 68 \\
\hline 1 OLDA & & 1 \\
\hline 1 Beni & set $C$ & 407 \\
\hline 1 Beni & & 181 \\
\hline 15 & & 337 \\
\hline 6 Dresden & & 330 \\
\hline 4 Portland & & 67 \\
\hline 1 OLDA & set $J$ & 1 \\
\hline 1 OLDA & set $B$ & 1 \\
\hline 1 OLDA & & 1 \\
\hline 1 Philadelphia & set $M$ & 1 \\
\hline 1 OLDA & set M & 1 \\
\hline 1 Philadelphia & set $\mathrm{L}$ & 1 \\
\hline 1 OLDA & set $\mathrm{L}$ & 1 \\
\hline 1 OLDA & set $J$ & 1 \\
\hline 1 OLDA & set $B$ & 1 \\
\hline 1 OLDA & set $B$ & 1 \\
\hline 1 OLDA & set $B$ & 1 \\
\hline 1 OLDA & set $B$ & 1 \\
\hline 1 OLDA & & 1 \\
\hline 1 Beni & & 42 \\
\hline 10 & set $\mathrm{H}$ & 247 \\
\hline 1 Knoxville & set $G$ & 146 \\
\hline 1 Knoxville & set $G$ & 146 \\
\hline 6 Chiccago & set $A$ & 424 \\
\hline 6 Chiccago & set $A$ & 424 \\
\hline 1 Philadelphia & & 1 \\
\hline 6 Chiccago & set $A$ & 424 \\
\hline 1 Beni & & 425 \\
\hline 1 OLDA & & 7 \\
\hline 1 Beni & & 15 \\
\hline 10 & set $\mathrm{E}$ & 245 \\
\hline 10 & set $\mathrm{E}$ & 245 \\
\hline 10 & set $\mathrm{E}$ & 245 \\
\hline 10 & set $\mathrm{E}$ & 245 \\
\hline 10 & set $F$ & 246 \\
\hline 10 & set $F$ & 246 \\
\hline 1 Benidorm & set $C$ & 426 \\
\hline 1 Benidorm & set C & 426 \\
\hline 1 Knoxville & & 9 \\
\hline 1 Philadelphia & set $D$ & 387 \\
\hline 1 Philadelphia & set $D$ & 387 \\
\hline 6 Chiccago & & 30 \\
\hline 1 Philadelphia & & 332 \\
\hline 1 Philadelphia & & 332 \\
\hline 1 Philadelphia & & 36 \\
\hline 10 & & 440 \\
\hline 1 OLDA & set $\mathrm{K}$ & 9 \\
\hline 1 Knoxville & set $\mathrm{K}$ & 9 \\
\hline 10 & set $\mathrm{H}$ & 248 \\
\hline 0 & & \\
\hline
\end{tabular}

Fig. 2. UMPGA dendrogram generated from the VET pattern (Dice similarity coefficient) computed with the BioNumerics software for 57 strains. - Variable genetic element present. ST, sequence type; Set A to M epidemiologically related strains (Table 1) 
Reference List

1. Diederen BM (2008) Legionella spp. and Legionnaires disease. J Infect 56:1-12

2. Fields BS, Benson RF, Besser RE (2002) Legionella and Legionnaires disease: 25 years of investigation. Clin Microbiol Rev 15:506-526

3. Helbig JH, Bernander S, Castellani PM, Etienne J, Gaia V, Lauwers S, Lindsay D, Lück PC, Marques T, Mentula S, Peeters MF, Pelaz C, Struelens M, Uldum SA, Wewalka G, Harrison TG (2002) Pan-European study on culture-proven Legionnaires disease: distribution of Legionella pneumophila serogroups and monoclonal subgroups. Eur J Clin Microbiol Infect Dis 21:710-716

4. Fry NK, Alexiou-Daniel S, Bangsborg JM, Bernander S, Castellani-Pastoris M, Etienne J, Forsblom B, Gaia V, Helbig JH, Lindsay D, Lück PC, Pelaz C, Uldum SA, Harrison TG (1999) A multicenter evaluation of genotyping methods for the epidemiologic typing of Legionella pneumophila serogroup 1: Results from a pan-European study. Clin Microbiol Infect 5:462-477

5. Bernander S, Jacobson K, Helbig JH, Lück PC, Lundholm M (2003) A hospitalassociated outbreak of Legionnaires disease caused by Legionella pneumophila serogroup 1 is characterized by stable genetic fingerprinting but variable monoclonal antibody patterns. J Clin Microbiol 41:2503-2508

6. Borchardt J, Helbig JH, Lück PC (2008) Occurrence and distribution of sequence types among Legionella pneumophila strains isolated from patients in Germany: common features and differences to other regions of the world. Eur J Clin Microbiol Infect Dis 27:29-36

7. Gaia V, Fry NK, Afshar B, Lück PC, Meugnier H, Etienne J, Peduzzi R, Harrison TG (2005) Consensus sequence-based scheme for epidemiological typing of clinical and environmental isolates of Legionella pneumophila. J Clin Microbiol 43:2047-2052

8. Ratzow S, Gaia V, Helbig JH, Fry NK, Lück PC (2007) Addition of neuA, the gene encoding $\mathrm{N}$-acylneuraminate cytidylyl transferase, increases the discriminatory ability of the consensus sequence-based scheme for typing Legionella pneumophila serogroup 1 strains. J Clin Microbiol 45:1965-1968

9. Afshar B, Fry NK, Bellamy W, Underwood AP, Harrison TG, Harrison TG (2007) External quality assessment of a DNA sequence-based scheme for epidemiological typing of Legionella pneumophila by an international network of laboratories. J Clin Microbiol 45:3251-3256

10. Cazalet C, Rusniok C, Brüggemann H, Zidane N, Magnier A, Ma L, Tichit M, Jarraud S, Bouchier C, Vandenesch F, Kunst F, Etienne J, Glaser P, Buchrieser C (2004) Evidence in the Legionella pneumophila genome for exploitation of host cell functions and high genome plasticity. Nat Genet 36:1165-1173

11. Chien M, Morozova I, Shi S, Sheng H, Chen J, Gomez SM, Asamani G, Hill K, Nuara J, Feder M, Rineer J, Greenberg JJ, Steshenko V, Park SH, Zhao B, Teplitskaya E, Edwards JR, Pampou S, Georghiou A, Chou IC, Iannuccilli W, Ulz ME, Kim DH, Geringer-Sameth A, Goldsberry C, Morozov P, Fischer SG, Segal G, Qu X, Rzhetsky A, Zhang P, Cayanis E, De Jong PJ, Ju J, Kalachikov S, Shuman HA, Russo JJ (2004) 
The genomic sequence of the accidental pathogen Legionella pneumophila. Science 305:1966-1968

12. Glöckner G, Albert-Weissenberger C, Weinmann E, Jacobi S, Schunder E, Steinert M, Hacker J, Heuner K (2008) Identification and characterization of a new conjugation/type IVA secretion system (trb/tra) of Legionella pneumophila Corby localized on two mobile genomic islands. International Journal of Medical Microbiology 298:411-428

13. Brassinga AK, Hiltz MF, Sisson GR, Morash MG, Hill N, Garduno E, Edelstein PH, Garduno RA, Hoffman PS (2003) A 65-kilobase pathogenicity island is unique to Philadelphia-1 strains of Legionella pneumophila. J Bacteriol 185:4630-4637

14. Heuner K, Bender-Beck L, Brand BC, Lück PC, Mann KH, Marre R, Ott M, Hacker J (1995) Cloning and genetic characterization of the flagellum subunit gene (flaA) of Legionella pneumophila serogroup 1. Infect Immun 63:2499-2507

15. Altschul SF, Madden TL, Schffer AA, Zhang J, Zhang Z, Miller W, Lipman DJ (1997) Gapped BLAST and PSI-BLAST: a new generation of protein database search programs. Nucleic Acids Res 25:3389-3402

16. Lück PC, Freier T, Steudel C, Knirel YA, Lüneberg E, Zhringer U, Helbig JH (2001) A point mutation in the active site of Legionella pneumophila $\mathrm{O}$-acetyltransferase results in modified lipopolysaccharide but does not influence virulence. Int J Med Microbiol 291:345-352

17. Wagner C, Krönert C, Lück PC, Jacobs E, Cianciotto NP, Helbig JH (2007) Random mutagenesis of Legionella pneumophila reveals genes associated with lipopolysaccharide synthesis and recognition by typing monoclonal antibodies. J Appl Microbiol 103:1975-1982

18. Huang B, Yuan Z, Heron BA, Gray BR, Eglezos S, Bates JR, Savill J (2006) Distribution of 19 major virulence genes in Legionella pneumophila serogroup 1 isolates from patients and water in Queensland, Australia. J Med Microbiol 55:993-997

19. Srinivasan U, Zhang L, France AM, Ghosh D, Shalaby W, Xie J, Marrs CF, Foxman B (2007) Probe hybridization array typing: a binary typing method for Escherichia coli. J Clin Microbiol 45:206-214

20. van Leeuwen W., Libregts C, Schalk M, Veuskens J, Verbrugh H, Van Belkum A. (2001) Binary typing of Staphylococcus aureus strains through reversed hybridization using digoxigenin-universal linkage system-labeled bacterial genomic DNA. J Clin Microbiol 39:328-331

21. Monecke S, Jatzwauk L, Weber S, Slickers P, Ehricht R (2008) DNA microarray-based genotyping of methicillin-resistant Staphylococcus aureus strains from Eastern Saxony. Clin Microbiol Infect 14:534-545 
1 Concise Article

2 Variable Genetic Element Typing: A quick method for

3 epidemiological subtyping of Legionella pneumophila

4

$5 \quad$ K. Pannier, K. Heuner, C. Lück

6

$7 \quad$ K. Pannier, C. Lück

8 Institute of Medical Microbiology and Hygiene, TU Dresden, 01307 Dresden, Germany

9

K. Heuner

11 Robert Koch-Institut, Nordufer 20, P26 - Nosocomial Infections of the Elderly, 13353 Berlin,

12 Germany

13

14

15

16 Corresponding author:

17 Christian. Lück

18 Institute of Medical Microbiology and Hygiene, TU Dresden,

19 Fiedlerstraße 42,

20 Dresden, D- 01307, Germany

21 E-mail: Christian.Lueck@tu-dresden.de

22

23 European Journal of Clinical Microbiology and Infectious Diseases, 2009 


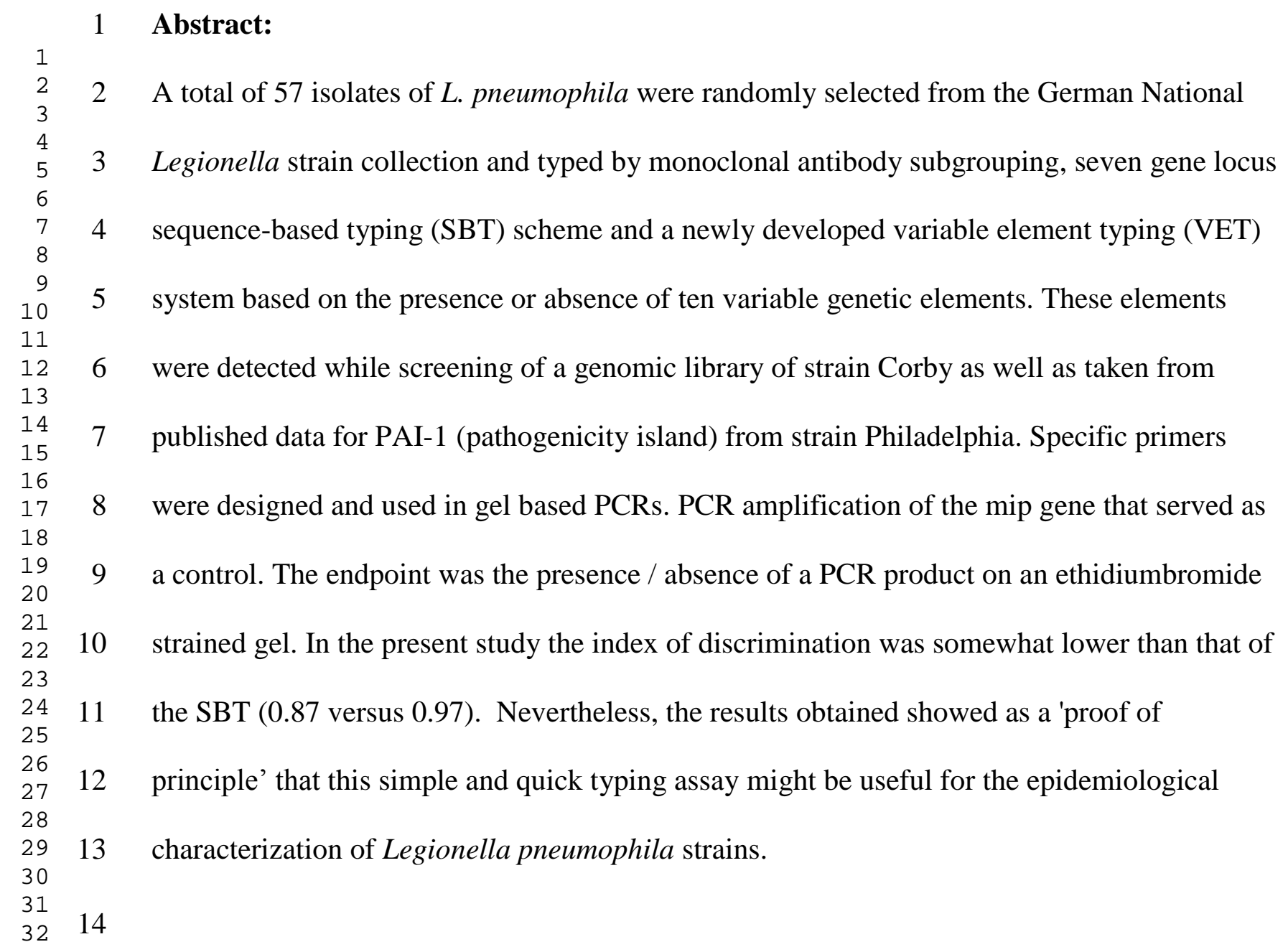




\section{Introduction}

2 Members of the genus Legionella are Gram-negative bacteria and normally occupy natural

3 aquatic environments where they survive as intracellular parasites of protozoa. Currently the

4 genus Legionella contains more than 50 species. Among them the species L. pneumophila is

5 the most frequent [1;2]. L. pneumophila serogroup (sg) 1 is the most common cause of

6 pneumonia occurring as sporadic or endemic disease that may be acquired from different

7 environmental sources [3]. For public health authorities it is essential to detect the source of

8 infection promptly by comparing clinical and environmental strains of L. pneumophila to

9 conduct decontamination measures and to prevent further cases. For this numerous

10 phenotypic and genotypic typing methods have been applied to the epidemiological typing of

11 L. pneumophila in the recent years [4]. This include monoclonal antibody (MAb)

12 subgrouping as a rapid screening method [3] and genotyping methods such as amplified

13 fragment length polymorphism (AFLP) [4], pulsed-field gel electrophoresis (PFGE) [4;5] and 14 sequence based typing [6-8]. In contrast to band based typing methods that are difficult to 15 standardize the developed sequence based typing (SBT) has the potential of excellent 16 typeability, interlaboratory reproducibility and epidemiologic concordance[9]. However the

17 SBT is rather expensive and might therefore not applicable to all strains and in all

18 laboratories. The specific gene content of each strain sequenced so far is constituted of 7-11\%

19 of gene specific to each genome [10-12]. In addition, variable elements/ pathogenicity island 20 are present in some strains [13].

21 The purpose of this study was to investigate the usefulness of a binary typing system (VET) 22 based on the presence/ absence of defined genetic elements by using gel based PCR. These 23 data were compared to the European Working Group on Legionella Infections (EWGLI) SBT 24 system. 


\section{Materials and Methods}

2 Legionella pneumophila strains. A total of 57 isolates of Legionella pneumophila were

3 randomly selected from the Dresden strain collection including the strains from which the

4 complete genomes are published. From strain Corby we used a panel of variants (mutants)

5 (EUL 135 to 139) that differ in their ability to multiply in amoebae and macrophages showed

6 different reactivity patterns with MAbs due to a mutation in a lipopolysaccharide synthesis

7 gene or that were resistant to rifampicin [7]. 40 strains were by definition unrelated to any

8 other isolates in the study, i.e. they were isolated from sporadic cases or if they were part of

9 an outbreak or cluster of legionellosis only one isolate was included in the analysis. 32

Variable element typing (VET). Target sequences for variable element typing were selected related strains (A to M). Some of them were indistinguishable by MAb subgrouping and SBT thus proving a transmission from the environmental source to the patient. Sets $\mathrm{C}$ and $\mathrm{H}$ differed in both markers, whereas sets $\mathrm{K}, \mathrm{L}$, and $\mathrm{M}$ were indistinguishable by genetic typing but expressed a different monoclonal subtype which is related to the deletion of the lag -1 gene [5] (Table 1).

\section{Serotyping and sequence based typing (SBT)}

Serological typing of $L$. pneumophila strains was performed by using the Dresden panel of monoclonal antibodies and the results were published previously [3;5;8]. Genotyping was performed by using the seven genes SBT [7;8]. Briefly, seven gene targets comprising flaA, pile, asd, mip, momp, proA and neuA were amplified by PCR and sequenced. The assignment of the sequence type (ST) was carried out by using SBT database or the online sequence type checker (http://www.hpa-bioinfotools.org.uk/legionella/php/sbt_query1.php ). 
1 modifications. Briefly, chromosomal DNA of L. pneumophila Corby (sg 1) was partially

2 digested with Sau3A. Fragments ranging from 1.0 to $4.0 \mathrm{~kb}$ were ligated into the BamHI

3 restriction site of vector pUC19 and transformed into E. coli DH5 $\alpha$. The DNA sequence of

4 the inserted Corby-DNA was determined by using standard $\mathrm{m} 13$ primers and primer walking.

5 Fragments that could not be aligned to the Philadelphia genome available at that time were

6 selected. Primer specific for these genetic elements were designed and used for typing (Table

7 2). For magA and traD sequences from the pathogenicity island [13] were used.

8 DNAs from L. pneumophila strains was prepared as for sequenced based typing [7]. The

9 VET- PCR were performed with 10pmol of each primer, $10 \mu 1$ of DNA-puffer, $5 \mathrm{U}$ of Gold-

10 Taq-Polymerase, $2 \mu 1$ DNA of each strain, and 200pmol of each dNTP at a final volumes of

$1150 \mu 1$. The annealing temperature was $58^{\circ} \mathrm{C}$. Gel electrophoresis was performed on $1 \%$

12 agarose gels. Gels were stained with ethidium bromide and analyzed under UV-light. PCR

13 products of the expected size were considered positive. At the beginning of these experiments

14 the specificity of the PCR products was confirmed by DNA sequencing [7]. In each run the

15 mip gene PCR of the SBT scheme were used as a positive control [7]. All VET PCR assays

16 were run at least twice.

17 The binary results (presence/absence of a PCR product) were recorded as a VET type and

18 compared to both standard methods (monoclonal antibodiy typing and SBT) (Table 1). These

19 binary data is offer the possibility to exchange these data between laboratories. 


\section{$1 \quad$ Results and Discussion}

2 The primary aim of this study was to prove the principle of VET as a genotyping method for

3 L. pneumophila. The primers developed and used in this study were aligned to the four

4 available complete genomes of L. pneumophila [10;11;12] by using the Basic Local

5 Alignment Search Tool (BLAST) available online (www.ncbi.nlm.nih.gov/BLAST) [15]. The

6 results of this search demonstrated that the binding sites of some primers were present some

7 of the four genomes. The investigation of the strains Philadelphia-1, Lens, Paris and Corby by

8 using the new VET-PCRs confirmed the expected results, i. e. all PCR product predicted by

9 the BLAST search could readily detected by using the ten VET-PCR assays. The variable strains from which complete sequenced genomes are available (Fig.1).

In addition, all five variants of the Corby strains that differ in the cultivability in amoebal hosts, point mutations in the $r p o \mathrm{~B}$ and the lag-1 genes showed the same VET results (data not shown) thus arguing that the markers detected in the VET are stable as were the SBT results [7].

For 57 of the strains investigated by using variable element typing (VET) results were obtained for 51. In six strains none of the elements could be detected by the current assays (Table 1). Since the positive control PCR using the mip primers of the SBT scheme gave positive results we are sure that the genetic elements or at least one of the primer binding sites were readily absent (or to less identical). From a total of 57 strains, 32 related strains were merged into 12 sets (A to M) (Table 1, Fig 2). From these sets of related strains the results of seven sets (A, B, D, E, F, G, and J) showed that related strains were indistinguishable by using for MAb typing, SBT and VET. For further two sets (C and H), the serotype/MAb subtype matched. However, SBT and VET of the environmental isolates were different from that of the patient's isolates. Vice versa three sets $(\mathrm{K}, \mathrm{L}$, and $\mathrm{M})$ showing the same genetic fingerprints by using SBT and VET were distinguishable by MAb typing. It has been shown 
1 previously that changes in genes involved in the LPS synthesis might change the reactivity

2 with MAbs. Thus the loss of the lag-1 gene coding for an O-acetyltransferase is responsible

3 for the switch from MAbtype Philadelphia/ Knoxville to the MAb type OLDA/Denver

$4 \quad[5 ; 16 ; 17]$.

5 Taking into account the moderate number of unrelated strains $(n=40)$ in our preliminary study

6 a moderate index of discrimination (IOD) of 0.87 was calculated. This is lower than that

7 published previously for SBT, but higher than that for MAb typing and AFLP [4;7].

8 The most frequently occurring VET-type $0,0,0,0,1,0,1,0,1,0$, was found in several strains

9 belonging to different MAb-types and but belonged all to ST 1. Interestingly, some strains

10 belonging to different serotypes were indistinguishable by VET (e.g., L02-298, L05-129)

11 (Table 1).

12 Due to the limited number of strains a general conclusion concerning the correlation between 13 these two genotyping methods is difficult. It is not surprising that a complete correlation does

14 not exist between both genotyping methods, since SBT detects variations in seven genes

15 belonging to the core genome whereas VET detects the absence or presence of variable

16 genetic elements like PAIs. Therefore, it is not surprising that some strains of sequence type

17 (ST) 1 investigated here, had a different VET-type.

18 SBT is a powerful tool for subtyping L. pneumophila strains and is currently the gold standard 19 within the European Working Group on Legionella Infections (EWGLI). Data obtained in 20 different countries can easily be compared and the European data base currently containing 21 hundreds of types can be used both in epidemiological investigations and to assess the risk 22 associated with environmental reservoirs that are contaminated with illness-associated strains. 23 However SBT is relatively expensive and therefore might not be applicable to all laboratories.

24 The aim of the present study was to evaluate an alternative rapid and cheep PCR approach 25 based on the absence or presence of defined genetic elements in different strains. A recent 26 study from Australia suggested that in all strains a similar repertoire of virulence gene exist 
1 [18]. In accordance with this study our results demonstrate that the presence of the $l v h$ locus,

2 the $t r b-1$ locus and the pathogenicity island I are variable in different strains. So far no

3 association of the presence of these genetic elements with the putative virulence i. e. the

4 origin of the strains could be demonstrated.

5 The usefulness of the VET approach as a technique for subtyping of strains belonging to

6 different serogroups of L. pneumophila was clearly demonstrated. The possibility to express

7 the data as binary codes allows the standardization and portability of these results as shows

8 for Staphylococcus aureus and Escherichia coli [19;20]. It remains an open question and

9 needs to be experimentally proven with a larger number of strains to whether the use of VET

10 is indeed suitable for the epidemiological subtyping of L. pneumophila. Finally, it must be

11 pointed out that these genetic elements might serve as candidates for a DNA chip based

12 typing system similar to that for St. aureus [21]. In summary, our current results are

13 encouraging. The VET scheme showed good typeability and discriminatory power for $L$.

14 pneumophila. However, further variable elements must be identified to increase both, the

15 typeability and the discriminatory potential.

16

17 Acknowledgements. Legionella-strains were kindly supplied by C. Aepinus (Würzburg), W.

18 Ehret (Augsburg), E. Halle (Berlin), Hohlfelder (Heidelberg), D. Jonas (Freiburg), W.

19 Matthys (Münster), R. Pfüller (Berlin), R. Marre (Ulm), C. Schoerner (Erlangen), K. Weist

20 (Berlin). We are grateful to Kerstin Lück and Jutta Paasche for technical assistance. 
Table 1. Legionella pneumophila strains

\begin{tabular}{|c|c|c|c|c|c|}
\hline Strain designation & $\begin{array}{c}\text { Serogroup, } \\
\text { Monoclonal } \\
\text { Subtype }\end{array}$ & $\begin{array}{l}\text { Sequence } \\
\text { Type } \\
\text { (ST) }\end{array}$ & $\begin{array}{l}\text { Allels profile of } \\
\text { flaA, pilE, asd, } \\
\text { mip, ompS, proA } \\
\text { and neuA }\end{array}$ & $\begin{array}{c}\text { Variable } \\
\text { element } \\
\text { typing VET }\end{array}$ & Origin of strains \\
\hline Lens & 1 Benidorm & 15 & $12,9,26,5,26,17,15$ & 0000101000 & Clinical isolate community acquired pneumonia, unrelated \\
\hline Augsburg-1 & 1 Benidorm & 42 & $4,7,11,3,11,12,9$ & 1111101101 & Clinical isolate community acquired pneumonia, unrelated \\
\hline Berlin 11 & 1 Benidorm & 425 & $2,10,3,15,9,4,11$ & 0000100010 & Clinical isolate nosocomial pneumonia, unrelated \\
\hline L02-287 & 1 Benidorm & 181 & $3,7,1,12,14,9,9$ & 1100001001 & Clinical isolate community acquired pneumonia, unrelated \\
\hline L02-298 & 1 Benidorm & 42 & $4,22,11,3,11,12,9$ & 0000101011 & Clinical isolate community acquired pneumonia, unrelated \\
\hline L04-412 & 1 Knoxville & 9 & $3,10,1,3,14,9,11$ & 0000000011 & Clinical isolate travel-associate pneumonia, unrelated \\
\hline Corby & 1 Knoxville & 51 & $6,10,15,28,9,14,6$ & 1111100011 & Clinical isolate community acquired pneumonia, unrelated \\
\hline L01-443 & 1 Knoxville & 9 & $3,10,1,3,14,9,11$ & 0111000011 & Clinical isolate nosocomial pneumonia, unrelated \\
\hline Berlin 4 & 1 OLDA & 1 & $1,4,3,1,1,1,1$ & 0101110010 & Clinical isolate nosocomial pneumonia, unrelated \\
\hline Charite $16297 / 2000$ & 1 OLDA & 1 & $1,4,3,1,1,1,1$ & 0110111010 & Clinical isolate community acquired pneumonia, unrelated \\
\hline Goettingen 12/98 & $1 \mathrm{OLDA}$ & 1 & $1,4,3,1,1,1,1$ & 0000111010 & Clinical isolate community acquired pneumonia, unrelated \\
\hline L03-610 & 1 OLDA & 7 & $1,4,3,1,1,1,6$ & 0000100010 & Clinical isolate community acquired pneumonia, unrelated \\
\hline L04-567 & 1 OLDA & 1 & $1,4,3,1,1,1,1$ & 0000101010 & Clinical isolate nosocomial pneumonia, unrelated \\
\hline Paris & 1 Philadelphia & 1 & $1,4,3,1,1,1,1$ & 0000001010 & Clinical isolate community acquired pneumonia, unrelated \\
\hline Philadelphia & 1 Philadelphia & 36 & $3,4,1,1,14,9,1$ & 0000011100 & Clinical isolate community acquired pneumonia, unrelated \\
\hline L04-280 & 1 Philadelphia & 23 & $2,3,9,10,2,1,6$ & 1111100010 & Clinical isolate travel-associate pneumonia, unrelated \\
\hline L02-034 & 10 & 440 & $3,10,1,28,14,9,1$ & 0000000000 & Clinical isolate nosocomial pneumonia, unrelated \\
\hline L02-303-1 & 15 & 337 & $10,22,7,28,16,18,6$ & 0100001001 & Clinical isolate nosocomial pneumonia, unrelated \\
\hline Heidelberg P9 & 4 Portland & 67 & $1,10,3,28,9,4,1$ & 0010011001 & Clinical isolate nosocomial pneumonia, unrelated \\
\hline W 01/1993 & 5 Cambridge & 245 & $1,10,3,13,9,4,1$ & 1111101000 & Environmental isolate, unrelated \\
\hline
\end{tabular}


Chicago 2

Finnl. 10

Heidelberg P1

L04-507

L04-506

W05-007

L04-597

L05-008

W04-952

W04-954

W04-956

Erl. 361/1

L04-541

W05-67-1

W05-67-4

L04-497

L97-229/1

L97-229/2

W04-989

W04-990
6 Chicago

6 Chicago

6 Dresden

1 Philadelphia

1 Philadelphia

6 Chicago

6 Chicago

6 Chicago

1 OLDA

1 OLDA

1 OLDA

1 OLDA

1 OLDA

1 Benidorm

1 Benidorm

1 Benidorm

1 Philadelphia

1 Philadelphia

10

10
$3,10,1,3,14,9,9$

$3,13,1,28,14,9,3$

7,10,17,3,4,11,3

$7,10,17,6,14,11,3$

$7,10,17,6,14,11,3$

7,10,17,3,13,14,9

$7,10,17,3,13,14,9$

$7,10,17,3,13,14,9$

$1,4,3,1,1,1,1$

$1,4,3,1,1,1,1$

$1,4,3,1,1,1,1$

$1,4,3,1,1,1,1$

$1,4,3,1,1,1,1$

$2,6,17,14,12,11,11$

$2,6,17,14,12,11,11$

$6,10,15,3,21,14,9$

$2,6,17,14,13,11,11$

$2,6,17,14,13,11,11$

$2,10,3,13,9,4,1$

$2,10,3,13,9,4,1$
0000110110 Clinical isolate nosocomial pneumonia, unrelated

1100111011 Clinical isolate community acquired pneumonia, unrelated 0001001001 Clinical isolate nosocomial pneumonia, unrelated

0000010100 Clinical isolate community acquired pneumonia, unrelated 0000010100 Clinical isolate community acquired pneumonia, unrelated

0000001010 Set A: Hospital water isolate

0000001010 Set A: Patients isolate nosocomial pneumonia

0000001010 Set A: Patients isolate nosocomial pneumonia

0000101010 Set B: Hospital water isolate

0000101010 Set B: Hospital water isolate

0000101010 Set B: Hospital water isolate

0000101010 Set B: Patients isolate nosocomial pneumonia

0000101010 Set B: Patients isolate nosocomial pneumonia

0000001000 Set C: Environmental isolate working place

0000001000 Set C: Environmental isolate working place

1110000000 Set C: Patients isolate community-acquired pneumonia

0000000110 Set D: Patients isolate community-acquired pneumonia

0000000110 Set D: Patients isolate community-acquired pneumonia

0000001000 Set E: Environmental isolate

0000001000 Set E: Environmental isolate 


$\begin{array}{llll}\text { L04-564 } & 10 & 245 & 2,10,3,13,9,4,1 \\ \text { L04-565 } & 10 & 245 & 2,10,3,13,9,4,1 \\ \text { W05-192 } & 10 & 246 & 2,10,3,28,9,4,11 \\ \text { W05-191 } & 10 & 246 & 2,10,3,28,9,4,11 \\ & & & \\ \text { Koper 1 } & 1 \text { Knoxville } & 146 & 1,10,18,10,2,1,6 \\ \text { L02-435 } & 1 \text { Knoxville } & 146 & 1,10,18,10,2,1,6 \\ & & & \\ \text { W05-174-1 } & 10 & 248 & 3,13,1,1,14,9,11 \\ \text { W05-177-1 } & 10 & 248 & 3,13,1,1,14,9,11 \\ \text { L05-129 } & 10 & 247 & 1,4,3,5,1,1,11 \\ & & & \\ \text { L01-127 } & 1 \text { OLDA } & 1 & 1,4,3,1,1,1,1 \\ \text { W01/1967 } & 1 \text { OLDA } & 1 & 1,4,3,1,1,1,1 \\ & & & \\ \text { Uppsala 3 } & 1 \text { Knoxville } & 9 & 3,10,1,3,14,9,11 \\ \text { Uppsala 10 } & 1 \text { OLDA } & 9 & 3,10,1,3,14,9,11 \\ & & & \\ \text { Uppsala 59 } & 1 \text { Philadelphia } & 1 & 1,4,3,1,1,1,1 \\ \text { Uppsala 60 } & 1 \text { OLDA } & 1 & 1,4,3,1,1,1,1 \\ \text { Uppsala 21 } & 1 \text { Philadelphia } & 1 & 1,4,3,1,1,1,1 \\ \text { Uppsala 22 } & 1 \text { OLDA } & 1 & 1,4,3,1,1,1,1\end{array}$

0000001000 Set E: Patients isolate community-acquired pneumonia 0000001000 Set E: Patients isolate community-acquired pneumonia

0000001000 Set F: Hospital water isolate

0000001000 Set F: Hospital water isolate

0010101011 Set G: Environmental isolate from a hotel

0010101011 Set G: Patients isolate travel-associated pneumonia

0000000000 Set H: Environmental isolate

0000000000 Set H: Environmental isolate

0000101011 Set H: Patients isolate community-acquired pneumonia

0000101010 Set J: Clinical isolate nosocomial pneumonia

0000101010 Set J: Environmental isolate

000000000 Set K: Clinical isolate nosocomial pneumonia

0000000000 Set K: Clinical isolate nosocomial pneumonia

0000101010 Set L: Clinical isolate nosocomial pneumonia

0000101010 Set L: Clinical isolate nosocomial pneumonia

0000101010 Set M: Clinical isolate nosocomial pneumonia

0000101010 Set M: Clinical isolate nosocomial pneumonia 
Table 2. Primers used for variable element typing (VET)

\begin{tabular}{|c|c|c|c|c|}
\hline NR & $\begin{array}{l}\text { Primer } \\
\text { designation }\end{array}$ & Sequence $\left(5^{\prime}-3^{\prime}\right)$ & $\begin{array}{l}\text { Size of the PCR } \\
\text { Product/ gene }\end{array}$ & Reference \\
\hline \multirow[t]{2}{*}{1} & TrbI1-F & TAA GGA GTG AGA CTG ATG AAT C & $1247 / t r b I 1$ & 12 \\
\hline & TrbI1-R & CAT TGC ACT CCT CCT GTA TCA AT & & \\
\hline \multirow[t]{2}{*}{2} & TraG1-F & TGG AGA CGG TCA ATG AGC TTG A & $1279 /$ traGl & 12 \\
\hline & TraG1-R & CAT ATC GCG AGC CGA TGC GCT G & & \\
\hline \multirow[t]{2}{*}{3} & MRR-F & AAT GAG AAG TCT TGG CTA TAC TTA & 835/lpc0219 & This study \\
\hline & MRR-R & GAT ATT CTC TAG CTT CGT TCG & & \\
\hline \multirow[t]{2}{*}{4} & $\mathrm{R} 33-\mathrm{F}$ & CGA CTA GGC GCT TAA TTC TTG & $830 / l p c 2786$ & This study \\
\hline & $\mathrm{R} 33-\mathrm{R}$ & ACG GAA CCA TTA AGA CAG GTT & & \\
\hline \multirow[t]{2}{*}{5} & F2-F & ACT GGC ATG AGC AAT CAG TAG & $1017 / p a c L$ & This study \\
\hline & F2-R & ATG ATC ACC TGT CAG GAC AGA & $(l p c 2098)$ & \\
\hline \multirow[t]{2}{*}{6} & TraD-F & GCT TAT CAT CAC TTG CCC TTT & $633 /$ traD & 13 \\
\hline & TraD-R & GCA GAG ATA CAC CAC CAA TCC GA & & \\
\hline \multirow[t]{2}{*}{7} & LvhB10-F & GCA ATC GGA CTC AGG TTG CTA & $710 / l v h B 10$ & 12 \\
\hline & LvhB10-R & CTG CCA AAG CGC TCG AAG AAA & & \\
\hline \multirow[t]{2}{*}{8} & MagA-F & CTC TAT CGC TAA CGC ACA AGG & 469/magA & 13 \\
\hline & MagA-R & CGT TGA AGT AGT TAG TGA AAG & & \\
\hline \multirow[t]{2}{*}{9} & $127-2-\mathrm{F}$ & AAG CGA TTA GCA TAT TAC GGT TTC & 127/pacL & This study \\
\hline & $127-2-\mathrm{R}$ & CAT TGA TGA GAA CAA CTG CTA TTA TAA & & \\
\hline \multirow[t]{2}{*}{10} & $298-\mathrm{F}$ & GAT CTT TTA TGG CTG TTG TTT GAG C & 298/lpc1159-60 & This study \\
\hline & 298-2-R & GCC GCC TAT TTT TCG CAC TTT A & & \\
\hline
\end{tabular}




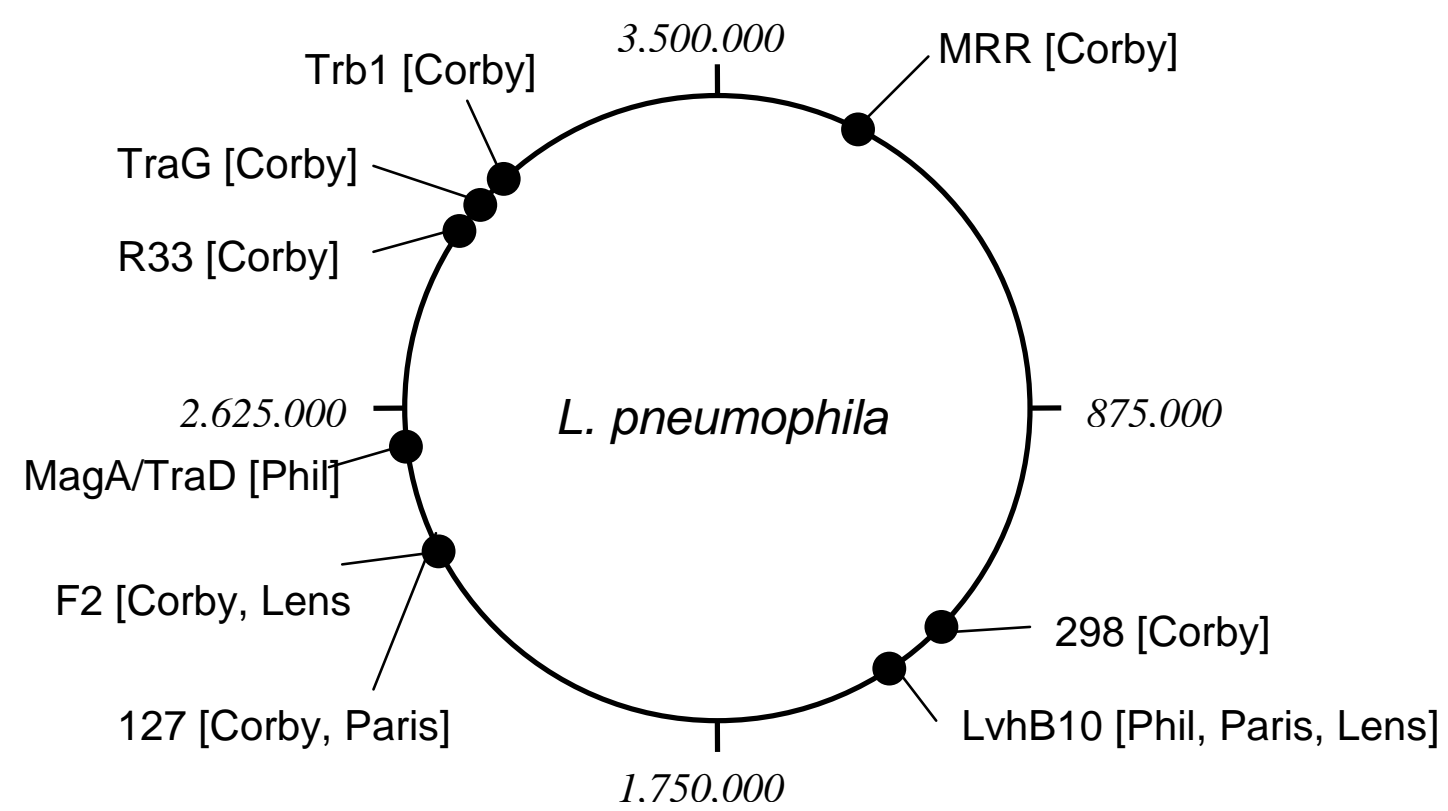

Fig. 1. Scheme of the location of the variable genetic elements in the four published genomes of L. pneumophila strains Philadelphia, Paris, Lens, and Corby 


\begin{tabular}{|c|c|c|}
\hline Sg/ MAb type & Set & ST \\
\hline 1 Knoxville & & 51 \\
\hline 1 Philadelphia & & 23 \\
\hline 1 Knoxville & & 9 \\
\hline 1 Beni & & 42 \\
\hline 5 Cambridge & & 245 \\
\hline 1 OLDA & & 1 \\
\hline 6 Chiccago & & 68 \\
\hline 1 OLDA & & 1 \\
\hline 1 Beni & set $C$ & 407 \\
\hline 1 Beni & & 181 \\
\hline 15 & & 337 \\
\hline 6 Dresden & & 330 \\
\hline 4 Portland & & 67 \\
\hline 1 OLDA & set J & 1 \\
\hline 1 OLDA & set $B$ & 1 \\
\hline 1 OLDA & & 1 \\
\hline 1 Philadelphia & set $M$ & 1 \\
\hline 1 OLDA & set $M$ & 1 \\
\hline 1 Philadelphia & set $\mathrm{L}$ & 1 \\
\hline 1 OLDA & set $\mathrm{L}$ & 1 \\
\hline 1 OLDA & set J & 1 \\
\hline 1 OLDA & set $B$ & 1 \\
\hline 1 OLDA & set $B$ & 1 \\
\hline 1 OLDA & set B & 1 \\
\hline 1 OLDA & set $B$ & 1 \\
\hline 1 OLDA & & 1 \\
\hline 1 Beni & & 42 \\
\hline 10 & set $\mathrm{H}$ & 247 \\
\hline 1 Knoxville & set $G$ & 146 \\
\hline 1 Knoxville & set $G$ & 146 \\
\hline 6 Chiccago & set $A$ & 424 \\
\hline 6 Chiccago & set $A$ & 424 \\
\hline 1 Philadelphia & & 1 \\
\hline 6 Chiccago & set $A$ & 424 \\
\hline 1 Beni & & 425 \\
\hline 1 OLDA & & 7 \\
\hline 1 Beni & & 15 \\
\hline 10 & set $E$ & 245 \\
\hline 10 & set $E$ & 245 \\
\hline 10 & set $E$ & 245 \\
\hline 10 & set $\mathrm{E}$ & 245 \\
\hline 10 & set $F$ & 246 \\
\hline 10 & set $F$ & 246 \\
\hline 1 Benidorm & set C & 426 \\
\hline 1 Benidorm & set C & 426 \\
\hline 1 Knoxville & & 9 \\
\hline 1 Philadelphia & set $D$ & 387 \\
\hline 1 Philadelphia & set $D$ & 387 \\
\hline 6 Chiccago & & 30 \\
\hline 1 Philadelphia & & 332 \\
\hline 1 Philadelphia & & 332 \\
\hline 1 Philadelphia & & 36 \\
\hline 10 & & 440 \\
\hline 1 OLDA & set $\mathrm{K}$ & 9 \\
\hline 1 Knoxville & set $\mathrm{K}$ & 9 \\
\hline 10 & set $\mathrm{H}$ & 248 \\
\hline 10 & set $\mathrm{H}$ & 248 \\
\hline
\end{tabular}

Fig. 2. UMPGA dendrogram generated from the VET pattern (Dice similarity coefficient) computed with the BioNumerics software for 57 strains. - Variable genetic element present. ST, sequence type; Set A to M epidemiologically related strains (Table 1) 


\section{Reference List}

1. Diederen BM (2008) Legionella spp. and Legionnaires disease. J Infect 56:1-12

2. Fields BS, Benson RF, Besser RE (2002) Legionella and Legionnaires disease: 25 years of investigation. Clin Microbiol Rev 15:506-526

3. Helbig JH, Bernander S, Castellani PM, Etienne J, Gaia V, Lauwers S, Lindsay D, Lück PC, Marques T, Mentula S, Peeters MF, Pelaz C, Struelens M, Uldum SA, Wewalka G, Harrison TG (2002) Pan-European study on culture-proven Legionnaires disease: distribution of Legionella pneumophila serogroups and monoclonal subgroups. Eur J Clin Microbiol Infect Dis 21:710-716

4. Fry NK, Alexiou-Daniel S, Bangsborg JM, Bernander S, Castellani-Pastoris M, Etienne J, Forsblom B, Gaia V, Helbig JH, Lindsay D, Lück PC, Pelaz C, Uldum SA, Harrison TG (1999) A multicenter evaluation of genotyping methods for the epidemiologic typing of Legionella pneumophila serogroup 1: Results from a pan-European study. Clin Microbiol Infect 5:462-477

5. Bernander S, Jacobson K, Helbig JH, Lück PC, Lundholm M (2003) A hospitalassociated outbreak of Legionnaires disease caused by Legionella pneumophila serogroup 1 is characterized by stable genetic fingerprinting but variable monoclonal antibody patterns. J Clin Microbiol 41:2503-2508

6. Borchardt J, Helbig JH, Lück PC (2008) Occurrence and distribution of sequence types among Legionella pneumophila strains isolated from patients in Germany: common features and differences to other regions of the world. Eur J Clin Microbiol Infect Dis 27:29-36

7. Gaia V, Fry NK, Afshar B, Lück PC, Meugnier H, Etienne J, Peduzzi R, Harrison TG (2005) Consensus sequence-based scheme for epidemiological typing of clinical and environmental isolates of Legionella pneumophila. J Clin Microbiol 43:2047-2052

8. Ratzow S, Gaia V, Helbig JH, Fry NK, Lück PC (2007) Addition of neuA, the gene encoding $\mathrm{N}$-acylneuraminate cytidylyl transferase, increases the discriminatory ability of the consensus sequence-based scheme for typing Legionella pneumophila serogroup 1 strains. J Clin Microbiol 45:1965-1968

9. Afshar B, Fry NK, Bellamy W, Underwood AP, Harrison TG, Harrison TG (2007) External quality assessment of a DNA sequence-based scheme for epidemiological typing of Legionella pneumophila by an international network of laboratories. J Clin Microbiol 45:3251-3256

10. Cazalet C, Rusniok C, Brüggemann H, Zidane N, Magnier A, Ma L, Tichit M, Jarraud S, Bouchier C, Vandenesch F, Kunst F, Etienne J, Glaser P, Buchrieser C (2004) Evidence in the Legionella pneumophila genome for exploitation of host cell functions and high genome plasticity. Nat Genet 36:1165-1173

11. Chien M, Morozova I, Shi S, Sheng H, Chen J, Gomez SM, Asamani G, Hill K, Nuara J, Feder M, Rineer J, Greenberg JJ, Steshenko V, Park SH, Zhao B, Teplitskaya E, Edwards JR, Pampou S, Georghiou A, Chou IC, Iannuccilli W, Ulz ME, Kim DH, Geringer-Sameth A, Goldsberry C, Morozov P, Fischer SG, Segal G, Qu X, Rzhetsky A, Zhang P, Cayanis E, De Jong PJ, Ju J, Kalachikov S, Shuman HA, Russo JJ (2004) 
The genomic sequence of the accidental pathogen Legionella pneumophila. Science 305:1966-1968

12. Glöckner G, Albert-Weissenberger C, Weinmann E, Jacobi S, Schunder E, Steinert M, Hacker J, Heuner K (2008) Identification and characterization of a new conjugation/type IVA secretion system (trb/tra) of Legionella pneumophila Corby localized on two mobile genomic islands. International Journal of Medical Microbiology 298:411-428

13. Brassinga AK, Hiltz MF, Sisson GR, Morash MG, Hill N, Garduno E, Edelstein PH, Garduno RA, Hoffman PS (2003) A 65-kilobase pathogenicity island is unique to Philadelphia-1 strains of Legionella pneumophila. J Bacteriol 185:4630-4637

14. Heuner K, Bender-Beck L, Brand BC, Lück PC, Mann KH, Marre R, Ott M, Hacker J (1995) Cloning and genetic characterization of the flagellum subunit gene (flaA) of Legionella pneumophila serogroup 1. Infect Immun 63:2499-2507

15. Altschul SF, Madden TL, Schffer AA, Zhang J, Zhang Z, Miller W, Lipman DJ (1997) Gapped BLAST and PSI-BLAST: a new generation of protein database search programs. Nucleic Acids Res 25:3389-3402

16. Lück PC, Freier T, Steudel C, Knirel YA, Lüneberg E, Zhringer U, Helbig JH (2001) A point mutation in the active site of Legionella pneumophila O-acetyltransferase results in modified lipopolysaccharide but does not influence virulence. Int J Med Microbiol 291:345-352

17. Wagner C, Krönert C, Lück PC, Jacobs E, Cianciotto NP, Helbig JH (2007) Random mutagenesis of Legionella pneumophila reveals genes associated with lipopolysaccharide synthesis and recognition by typing monoclonal antibodies. J Appl Microbiol 103:1975-1982

18. Huang B, Yuan Z, Heron BA, Gray BR, Eglezos S, Bates JR, Savill J (2006) Distribution of 19 major virulence genes in Legionella pneumophila serogroup 1 isolates from patients and water in Queensland, Australia. J Med Microbiol 55:993-997

19. Srinivasan U, Zhang L, France AM, Ghosh D, Shalaby W, Xie J, Marrs CF, Foxman B (2007) Probe hybridization array typing: a binary typing method for Escherichia coli. J Clin Microbiol 45:206-214

20. van Leeuwen W., Libregts C, Schalk M, Veuskens J, Verbrugh H, Van Belkum A. (2001) Binary typing of Staphylococcus aureus strains through reversed hybridization using digoxigenin-universal linkage system-labeled bacterial genomic DNA. J Clin Microbiol 39:328-331

21. Monecke S, Jatzwauk L, Weber S, Slickers P, Ehricht R (2008) DNA microarray-based genotyping of methicillin-resistant Staphylococcus aureus strains from Eastern Saxony. Clin Microbiol Infect 14:534-545 
Statements to the reviewer's comments

Reviewer \#1: This is a clear, and mainly well-presented, description of a binary typing method for Legionella pneumophila based on presence or absence of ten defined genetic elements. I have only two minor comments:

Since the number and diversity of the isolates examined is quite small and restricted in range, this study could not be described as an evaluation of the method but rather a 'proof of principle'. As such the last line of the abstract '. proved the usefulness..' does not seem to be supported by the data presented.

CL: The abstract has been changed: Now the phrase is

"The results obtained showed that this simple and quick typing assay might be useful for the epidemiological characterization of Legionella pneumophila strains".

It also has been changed at the first lane in the results and discussion section.

Perhaps it should be moderated a little to reflect the fact that this is a very preliminary evaluation. Also as written the abstract is not very informative. Since this is usually the only bit of a paper that most readers attend to, it might be helpful to add some data (even if it is only to say how many elements are looked at and that endpoint is presence/absence of a PCR product on a gel).

CL: The abstract was rewritten to clarify the points raised.

I may have missed something, but to me the VET-index seems an overly complex way of expressing binary data. Rather than group the ten 'scored' elements into three sets of three plus a singleton, to achieve a four digit index (the last digit of which can only ever be 0 or 1 ), why do the authors not leave it as a binary number? If writing a ten digit number is considered to be burdensome, or prone to transcription error, then simply express it as a decimal giving a single value for each profile running from $0-1,023$ ?

CL: I agree with the reviewer that the introduction of an index is too complicated. Keeping in mind the relatively low number of strains this seems to be not indicated. One might considerer this again when a larger number of strains have been typed. The text and table 1 were changed accordingly. 